\title{
Interaction of Mycobacterium tuberculosis CYP130 with Heterocyclic Arylamines ${ }^{*[5}$
}

Received for publication, May 6, 2009, and in revised form, June 16, 2009 Published, JBC Papers in Press, July 15, 2009, DOI 10.1074/jbc.M109.017632

\author{
Larissa M. Podust ${ }^{\ddagger}$, Hugues Ouellet ${ }^{\ddagger}$, Jens P. von Kries ${ }^{\S}$, and Paul R. Ortiz de Montellano ${ }^{\ddagger 1}$ \\ From the ${ }^{\ddagger}$ Department of Pharmaceutical Chemistry, University of California, San Francisco, California 94158 and the ${ }^{\S}$ Screening \\ Unit, Leibniz Institute for Molecular Pharmacology, Berlin 13125, Germany
}

The Mycobacterium tuberculosis $\mathbf{P} 450$ enzymes are of interest for their pharmacological development potential, as evidenced by their susceptibility to inhibition by antifungal azole drugs that normally target sterol $14 \alpha$-demethylase (CYP51). Although antifungal azoles show promise, direct screening of compounds against $M$. tuberculosis $\mathbf{P} 450$ enzymes may identify novel, more potent, and selective inhibitory scaffolds. Here we report that CYP130 from M. tuberculosis has a natural propensity to bind primary arylamines with particular chemical architectures. These compounds were identified via a high throughput screen of CYP130 with a library of synthetic organic molecules. As revealed by subsequent $\mathrm{X}$-ray structure analysis, selected compounds bind in the active site by Fe-coordination and hydrogen bonding of the arylamine group to the carbonyl oxygen of $\mathrm{Gly}^{243}$. As evidenced by the binding of structural analogs, the primary arylamine group is indispensable, but synergism due to hydrophobic contacts between the rest of the molecule and protein amino acid residues is responsible for a binding affinity comparable with that of the antifungal azole drugs. The topology of the CYP130 active site favors angular coordination of the arylamine group over the orthogonal coordination of azoles. Upon substitution of $\mathrm{Gly}^{243}$ by an alanine, the binding mode of azoles and some arylamines reverted from type II to type I because of hydrophobic and steric interactions with the alanine side chain. We suggest a role for the conserved Ala(Gly) ${ }^{243}$ Gly $^{244}$ motif in the I-helix in modulating both the binding affinity of the axial water ligand and the ligand selectivity of cytochrome P450 enzymes.

CYP130 is one of the 20 Mycobacterium tuberculosis cytochrome P450 (P450, CYP) $)^{2}$ enzymes and is one of three (CYP51, CYP121, and CYP130) that have been studied as individually expressed proteins at the structural level. Evidence has accu-

* This work was supported, in whole or in part, by National Institutes of Health RO1 Grants Al07824 and GM25515 (to P. O. d. M.) and GM078553 (to L. M. P.). This work was also supported by Grants BIO/0312992A (to J. P. K.) from the X-Mtb Consortium Bundesministerium fuer Bildung und Forschung/Projekttraeger Juelich (BMBF/PTJ).

5 The on-line version of this article (available at http://www.jbc.org) contains supplemental Figs. S1-S4 and Tables S1 and S2.

The atomic coordinates and structure factors (codes 2WH8, 2WGY, and 2WHF) have been deposited in the Protein Data Bank, Research Collaboratory for Structural Bioinformatics, Rutgers University, New Brunswick, NJ (http://www.rcsb.org/).

${ }^{1}$ To whom correspondence should be addressed. Tel.: 415-476-2903; E-mail: ortiz@cgl.ucsf.edu.

${ }^{2}$ The abbreviations used are: CYP or P450, cytochrome P450; PDB, Protein Data Bank. mulated for the importance of $M$. tuberculosis P450 enzymes in virulence (CYP132) (1), host infection (CYP125) (2), and pathogen viability (CYP128, CYP121) (3, 4), although neither their exact biological functions nor any of the endogenous substrates upon which these enzymes operate have yet been established. However, it has recently been shown in vitro that CYP121 catalyzes a $\mathrm{C}-\mathrm{C}$ coupling reaction between two tyrosine groups (5). CYP130 is absent from the genome of Mycobacterium bovis, suggesting that it might play specific role(s) in the infection of the human host and thus constitute a potential therapeutic target.

The potential of M. tuberculosis P450 enzymes for pharmacologic development was initially suggested by their susceptibility to inhibition by antifungal azole drugs such as fluconazole, econazole, and clotrimazole. These drugs block sterol $14 \alpha$-demethylase CYP51 in fungi (6), tightly bind to M. tuberculosis $\mathrm{P} 450$ proteins $(7,8)$, and display inhibitory potential against latent and multidrug-resistant forms of tuberculosis both in vitro and in tuberculosis-infected mice (9-14).

The substantial differences between fungal CYP51 and the potential P450 targets in microbial pathogens, including $M$. $t u$ berculosis, suggest that the direct screening of compounds against $M$. tuberculosis CYP enzymes could identify novel inhibitory scaffolds that are more potent and selective than antifungal drugs. Structurally characterized screening targets are advantageous, as the already defined purification and crystallization protocols can be applied to obtain co-crystal structures and to elucidate the binding modes of screening hits. This approach has been successfully applied to CYP51, resulting in identification of novel inhibitory scaffolds for CYP51 therapeutic targets $(15,16)$.

Toward this goal, the property of P450 enzymes to shift the ferric heme iron Soret band on ligand binding (17) provides an experimental platform for high throughput screening of compound libraries to select chemotypes with high binding affinities for the target. Expulsion of the heme iron axial water ligand from the Fe-coordination sphere by the incoming substrate followed by transition of the ferric heme from the low-spin hexacoordinated to the high-spin pentacoordinated state characterize type I spectral shifts and are a prerequisite for P450 catalytic activity. Replacement of a weak axial ligand, the water molecule, with a stronger one possessing a nitrogen-containing aliphatic or aromatic group coordinating to the heme iron characterizes type II spectral shifts.

To find new high affinity ligands of CYP130, a commercial library of 20,000 small organic molecules comprising a large selection of molecular scaffolds was screened against the 
enzyme. In contrast to the results with CYP51, no type I binding hits were identified. Screening produced about a dozen structurally diverse type II hits that were unexpectedly devoid of the usual aromatic nitrogen atoms readily accessible for axial coordination of the heme iron, suggesting an alternative coordination mode. High resolution $\mathrm{x}$-ray structure analysis determined that two compounds coordinated to the heme iron via a primary arylamine group, providing the first structural evidence on P450-heterocyclic arylamine interactions.

\section{EXPERIMENTAL PROCEDURES}

Materials and Reagents-The CYP130 ${ }_{\mathrm{G} 243 \mathrm{~A}}$ mutant is a new protein construct. Amino acid substitution was carried out using the QuikChange site-directed mutagenesis kit (Stratagene) following the recommended protocol. The cloned M. tuberculosis cyp130 gene was used as a template with the complementary oligonucleotide primers 5'-TTCACCATGGTCACCGCCGGCAACGACACCGTC-3' and 5' -GACGGTGTCGTTGCCGGCGGTGACCATGGTGAA-3'. The correctness of the DNA construct was confirmed by sequencing. Wild-type recombinant CYP130 and its G243A mutant were purified as described previously (8). CYP142 was prepared similarly to CYP130.

Both the library and most of the separately purchased compounds, including compound 2, (5-amino-2-\{4-[(4-aminophenyl)sulfanyl]phenyl $\}-1 H$-isoindole-1,3(2H)-dione), and compound 4, (1-(3-methylphenyl)-1H-benzimidazole-5-amine), were from ChemDiv (San Diego). Compound 5 (4-(2,3bis(3,4,5,6-tetrahydropyridin-2-yl)quinoxalin-6-yloxy)aniline was from ChemBridge (San Diego). The nitro analog of compound 2, 2-(4-(4-nitrophenylthio)phenyl)isoindole-1,3-dione was from Asinex USA (Winston-Salem, NC). Aniline, benzylamine, econazole, glucose, Aspergillus niger glucose oxidase, bovine liver catalase, spinach ferredoxin, spinach NADP+ferredoxin reductase, and NADPH were from Sigma-Aldrich.

Automated Library Screening and Data Analysis-Screening of the FMP-20 compound library (ChemDiv) containing 20,000 small organic molecules was performed as described elsewhere (15). Briefly, library compounds were solubilized at a stock concentration of $10 \mathrm{~mm}$ in $\mathrm{Me}_{2} \mathrm{SO}$ and distributed in $0.4-\mu \mathrm{l}$ aliquots in 384-well microtiter plates that were stored sealed with aluminum foil at $-20^{\circ} \mathrm{C}$. Prior to use, $40 \mu \mathrm{l}$ of $50 \mathrm{~mm}$ Tris- $\mathrm{HCl}$, $\mathrm{pH} 7.5$, containing $10 \%$ glycerol was added to each well to achieve a concentration of $100 \mu \mathrm{M}$ and to reduce the $\mathrm{Me}_{2} \mathrm{SO}$ concentration to $1 \%$. The plates were subsequently incubated at $37^{\circ} \mathrm{C}$ for $15 \mathrm{~min}$ followed by $5 \mathrm{~min}$ of sonication using an ultrasonic water bath (Transsonic 460, Elma) to allow the suspensions of the most hydrophobic compounds to solubilize. Wells were not inspected visually for precipitates or cloudiness upon dilution. To account for the optical properties of the test compounds, compound-specific changes of absorption spectra $(310-450 \mathrm{~nm})$ were recorded before and after $10 \mu \mathrm{l}$ of $4 \mu \mathrm{M}$ wild-type CYP130 was added to each well containing either test or reference compounds in the same buffer. Econazole at two different concentrations, 10 and $50 \mu \mathrm{M}$, was used as reference for the type II binding mode. No reference compounds for type I binding were available for CYP130.
Absorption data were exported to an EXCEL spreadsheet, and background spectra obtained for compounds in the absence of protein, as well as protein in the absence of compound, were subtracted. Absorption values were plotted against wavelength. Curves obtained for the reference were superimposed on the curves obtained for the test compounds. Novel ligands were identified by curve similarity.

Hit validation in the automated regime was performed at 10-fold increased CYP130 target concentration (from 1 to 10 $\mu \mathrm{M})$ to achieve better signal-to-noise ratio, and 10-fold reduced (from 100 to $10 \mu \mathrm{M}$ ) concentration of the hit compounds. Hits that demonstrated absorption difference spectra compatible with the econazole reference even at the lowest concentration passed the validation test.

Spectroscopic Binding Assays -All ligand binding assays were performed by spectrophotometric titration in $50 \mathrm{~mm}$ potassium phosphate buffer, $\mathrm{pH} 7.4$, containing $0.1 \mathrm{~mm}$ EDTA using a Cary UV-visible scanning spectrophotometer (Varian). Stock solutions of the ligands were prepared in $\mathrm{Me}_{2} \mathrm{SO}$. To account for the absorbance of the tested compounds, $1 \mathrm{ml}$ of protein $(2.5$ $\mu \mathrm{M})$ in buffer was placed in the first chamber of two split cuvettes, and $1 \mathrm{ml}$ of buffer was placed in the second chamber. After background scanning, equal volumes $(0.25-1.0 \mu \mathrm{l})$ of ligand solution were titrated into both the second chamber of the reference cuvette containing only buffer and the first chamber of the sample cuvette containing the protein. The same volume of $\mathrm{Me}_{2} \mathrm{SO}$ was added into the alternate chambers to correct for organic solvent effects. Difference spectra were recorded from 300 to $500 \mathrm{~nm}$. To determine the $K_{D}$ values, titration data points were fitted to either the rectangular hyperbola (Equation 1), the quadratic hyperbola (Equation 2), or the Hill equation (Equation 3) using the Kaleidagraph software (Synergy). In all equations, $A_{\text {obs }}$ is the absorption shift determined at any ligand concentration; $A_{\max }$ is the maximal absorption shift obtained at saturation; $K_{D}$ is the apparent dissociation constant for the inhibitor-enzyme complex; $E_{t}$ is the total enzyme concentration used; $\mathrm{S}$ is the ligand concentration; and $n$ is a Hill coefficient, a measure of cooperativity.

$$
\begin{gathered}
A_{\mathrm{obs}}=A_{\max }\left(\mathrm{S} /\left(K_{D}+\mathrm{S}\right)\right) \\
A_{\mathrm{obs}}=\left(\left(A_{\mathrm{max}} /\left(2 \times E_{t}\right)\right)\left(\mathrm{S}+E_{t}+K_{D}\right)\right)-\left(\mathrm{S}+E_{t}+K_{D}\right)^{2}-(4 \times \mathrm{S} \\
\left.\times E_{t}\right)^{0.5} \\
A_{\mathrm{obs}}=A_{\max }\left(\mathrm{S}^{n} /\left(K_{D}^{n}+\mathrm{S}^{n}\right)\right)
\end{gathered}
$$

Anaerobic Conditions - To reproducibly maintain satisfactorily strict anaerobic conditions, when required, an enzymatic $\mathrm{O}_{2}$-scavenging system consisting of 2 units $/ \mathrm{ml}$ glucose oxidase, $10 \mathrm{~mm}$ glucose, and 130 units $/ \mathrm{ml}$ catalase was added to protein samples.

Crystallization, Data Collection, and Structure Determination-Compounds $\mathbf{2 - 5}$ were used to screen crystallization conditions using commercial high throughput screening kits available in deep-well format (Hampton Research), a nanoliter drop-setting Mosquito robot (TTP LabTech) operating with 96-well plates, and a hanging drop crystallization protocol. Optimization of conditions was carried out manually in 24-well 
Role of Ala(Gly)-Gly Motif in the I-Helix

TABLE 1

Crystallographic data and statistics

Numbers in parentheses correspond to the highest resolution shell. r.m.s., root mean square; NA, not applicable.

\begin{tabular}{|c|c|c|c|}
\hline & CYP130-compound 2 & CYP130-compound 4 & $\mathrm{CYP} 130_{\mathrm{G} 243 \mathrm{~A}}$ ligand-free \\
\hline PDB ID code & 2WH8 & $2 \mathrm{WHF}$ & 2WGY \\
\hline \multicolumn{4}{|l|}{ Data collection } \\
\hline Wavelength $(\AA)$ & 1.11587 & 1.11587 & 1.11587 \\
\hline Resolution $(\AA)$ & 1.70 & 1.58 & 1.50 \\
\hline Unique reflections & 164,200 & 51,244 & 59,681 \\
\hline Redundancy & $1.8(1.7)^{a}$ & $3.6(3.4)$ & $3.5(2.5)$ \\
\hline Completeness (\%) & $96.0(93.1)$ & $100.0(99.6)$ & $97.0(78.2)$ \\
\hline Space group & P1 & $\mathrm{C} 2$ & $\mathrm{C} 2$ \\
\hline \multicolumn{4}{|l|}{ Cell dimensions } \\
\hline$a, b, c(\AA)$ & $53.7,84.9,90.2$ & $160.9,53.9,44.0$ & $160.2,54.1,43.3$ \\
\hline$\alpha, \beta, \gamma\left(\left(^{\circ}\right)\right.$ & $96.6,90.0,108.4$ & $90.0,96.7,90.0$ & $90.0,96.2,90$ \\
\hline Molecules in asymmetric unit & 4 & 1 & 1 \\
\hline Solvent content (\%) & 41.2 & 39.3 & 41.2 \\
\hline$R_{\mathrm{sym}}{ }^{a}(\%)$ & $3.4(33.1)$ & $3.7(44.7)$ & $3.4(12.0)$ \\
\hline$I / \sigma$ & $19.9(2.1)$ & $39.7(3.1)$ & $45.3(9.5)$ \\
\hline \multicolumn{4}{|l|}{ Refinement } \\
\hline Reflections used in refinement & 142,540 & 54,902 & 54,979 \\
\hline$R_{\text {cryst }}\left(R_{\text {free }}\right)^{b}(\%)$ & $19.9 / 25.9$ & $19.6 / 23.9$ & $18.3 / 21.3$ \\
\hline No. of atoms & 13,226 & 3,319 & 3,586 \\
\hline Protein & 12,098 & 3,078 & 3,180 \\
\hline Heme & 172 & 43 & 43 \\
\hline Ligand & 104 & 34 & NA \\
\hline Water/solvent & $851 / 0$ & $164 / 0$ & $323 / 40$ \\
\hline Wilson plot B-values $\left(\AA^{2}\right)$ & 21.2 & 23.9 & 16.1 \\
\hline Mean B-factor $\left(\AA^{2}\right)$ & 22.2 & 25.1 & 16.6 \\
\hline Protein $\left(\AA^{2}\right)$ & $22.5^{d}$ & 24.8 & 15.7 \\
\hline Heme $\left(\AA^{2}\right)$ & $11.9^{d}$ & 15.7 & 10.1 \\
\hline Ligand $\left(\AA^{2}\right)$ & $32.5^{d}$ & 4-1: $38.0 ; 4-2: 43.7$ & NA \\
\hline Water $\left(\AA^{2}\right)$ & 28.4 & 30.0 & 23.9 \\
\hline \multicolumn{4}{|l|}{ r.m.s. deviations } \\
\hline Bond length $(\AA)$ & 0.019 & 0.015 & 0.009 \\
\hline Bond angles $\left({ }^{\circ}\right)$ & 1.8 & 1.7 & 1.2 \\
\hline Ramachandran ${ }^{c}(\%)$ & $89.4 / 10.0 / 0.2 / 0.3^{d}$ & $89.2 / 10.5 / 0.3 / 0.0$ & $90.7 / 9.0 / 0.3 / 0.0$ \\
\hline
\end{tabular}

${ }^{a} R_{\text {sym }}=\Sigma\left|I_{i}-\langle I\rangle\right| / \Sigma I_{i}$, where $I_{i}$ is the intensity of the $i^{\text {th }}$ observation and $\langle I\rangle$ is the mean intensity of reflection.

${ }^{b} R_{\text {cryst }}=\Sigma|| F_{o}|-| F_{c}|/ \Sigma| F_{o} \mid$, calculated with the working reflection set. $R_{\text {free }}$ is the same as $R_{\text {cryst }}$ but calculated with the reserved reflection set.

${ }^{c}$ PROCHECK program (32), portions of the protein residues in most favored/additional allowed/generously allowed/disallowed regions.

${ }^{d}$ Averaged for chain A only.

plates. The protein was from $0.85 \mathrm{~mm}$ frozen stock in $20 \mathrm{~mm}$ Tris- $\mathrm{HCl}, \mathrm{pH} 7.5,200 \mathrm{~mm} \mathrm{NaCl}$, and $0.5 \mathrm{~mm}$ EDTA. Prior to crystallization, the protein was diluted to $0.1-0.2 \mathrm{~mm}$ by mixing with the compound dissolved at $2 \mathrm{~mm}$ in $10 \mathrm{~mm}$ Tris- $\mathrm{HCl}, \mathrm{pH}$ 7.5 .

Crystals of the CYP130-compound $\mathbf{2}$ complex grew in $12-16 \%$ polyethylene glycol 4000, 0.1 M sodium acetate, $\mathrm{pH} 5.0$, $200 \mathrm{~mm}$ ammonium sulfate, and 1\% isopropyl alcohol. Crystals of the CYP130-compound 4 complex grew in 18-22\% polyethylene glycol 4000, 0.1 M sodium acetate, pH 5.5, $200 \mathrm{~mm}$ ammonium sulfate, and $2 \%$ isopropyl alcohol. Crystals of the CYP130 ${ }_{\text {G243A }}$ mutant were obtained from $1.5 \mathrm{M}$ ammonium sulfate, $0.1 \mathrm{M}$ citric acid, pH 5.2, and 3\% isopropyl alcohol. Prior to data collection, the crystals were cryoprotected by plunging them into a drop of reservoir solution supplemented with $18 \%$ ethylene glycol and flash-frozen in liquid nitrogen. Diffraction data were collected at $100-110 \mathrm{~K}$ at Beamline 8.3.1, Advanced Light Source, Lawrence Berkeley National Laboratory. Data indexing, integration, and scaling were conducted using MOSFLM (18) and the HKL2000 software suite (19). Crystal structures were determined by molecular replacement using the atomic coordinates of the non-ligand-bound CYP130 (PDB ID code: 2UUQ) as a search model (Table 1).

\section{RESULTS}

Identification of Binding Hits-Automated screening of the library of organic molecules against the M.tuberculosis
CYP130 target resulted in about a dozen type II, but no type I, binding hits (supplemental Fig. S1A). The absence of type I hits is consistent with our previous observation that none of the compounds tested thus far could shift the CYP130 wild-type form into the pentacoordinated high-spin state that is a prerequisite for catalytic activity. Thus, no catalytic activity of any kind has so far been observed for CYP130. Most type II screen hits identified in these studies were heterocyclic arylamines with an overall chemical architecture that differs from that of the antifungal azoles. Remarkably, none of the top compounds contained a nitrogen atom within an aromatic heterocyclic ring in a position readily accessible for axial coordination to the heme iron.

Spectrophotometric Characterization of the Complexes-The electronic absorption spectra of ferric CYP130 in complex with compound 2 (Fig. 1 and supplemental Table S1) and compounds 4 and 5 (supplemental Fig. S2 and Table S1) exhibit red-shifted Soret bands, confirming the type II nature of these ligands. Because of the previously observed instability of the ferrous ligand-free form (20), it was impossible to monitor direct addition of the ligands to ferrous CYP130. Instead, we reduced the ferric complexes anaerobically using sodium dithionite. Of the three compounds tested, only compound 2 appeared to form a stable ferrous hexacoordinate complex. Indeed, a new species with very distinct features at 445.0, 527.8, and $557.6 \mathrm{~nm}$ was slowly formed (Fig. 1). Bubbling of CO into the solution after $60 \mathrm{~min}$ resulted in very slow conversion to the 
A

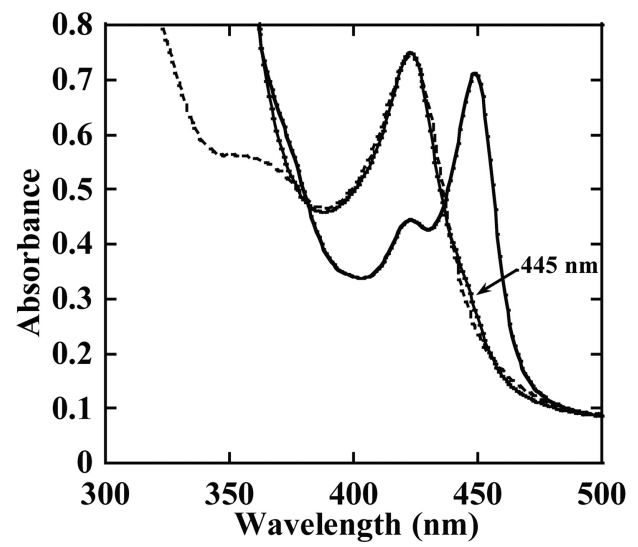

B

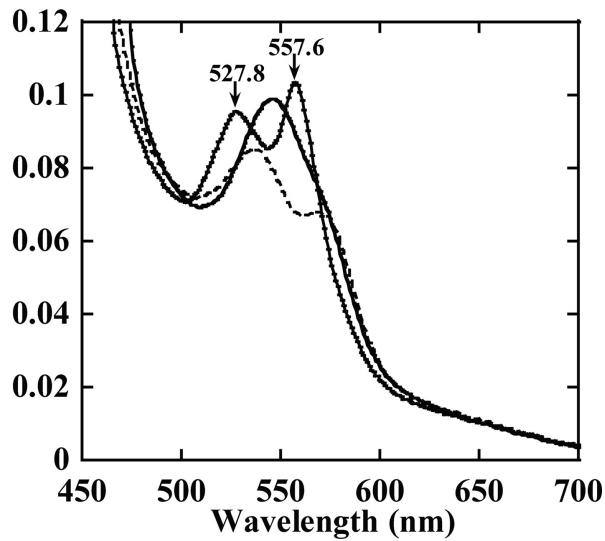

FIGURE 1. Electronic absorption spectra of the CYP130-compound 2 complex. The Soret $(A)$ and enlarged visible $(B)$ regions are shown. The ferric complex (dashed trace) was reduced to a stable ferrous form (thin trace). Once reduction was complete ( $~ 60 \mathrm{~min})$, CO was bubbled for $30 \mathrm{~s}$, and spectra were collected until equilibrium was reached (thick trace). The position of band maxima and their respective extinction coefficient values are summarized in the supporting material (supplemental Table S1). The spectra were recorded at the protein concentration of $5 \mu \mathrm{m}$ in $100 \mathrm{~mm}$ potassium phosphate buffer, pH 7.4, containing $0.1 \mathrm{~mm}$ EDTA, and compound $\mathbf{2}$ was added at a saturating concentration of $25 \mu \mathrm{m}$.

A

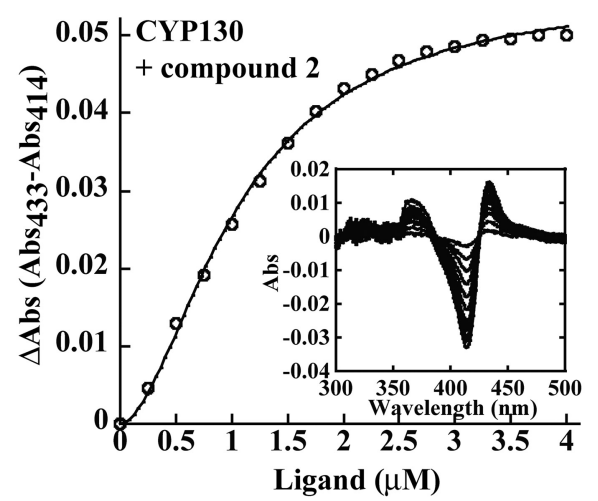

C

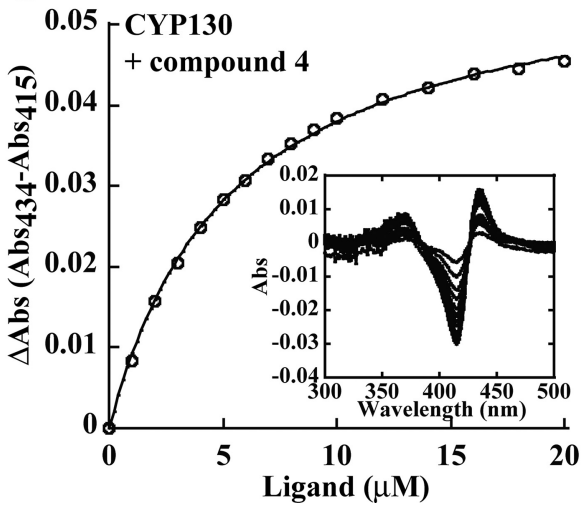

B

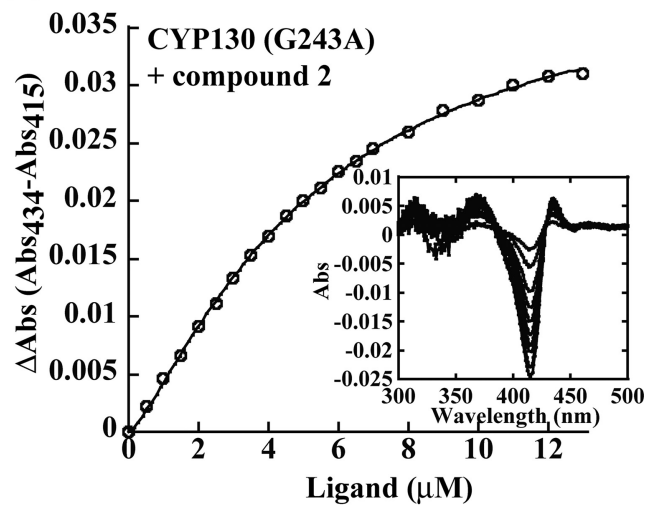

D

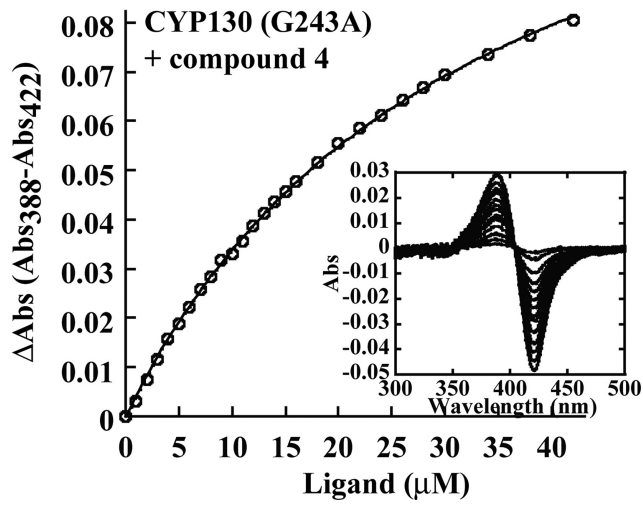

FIGURE 2. Binding of heterocyclic arylamines to $M$. tuberculosis CYP130 and CYP130 $_{\mathrm{G} 243 \mathrm{~A}}$ mutant. The concentration dependence of compound $\mathbf{2}(A$ and $B)$ and compound $\mathbf{4}(C$ and $D)$ binding deduced from the difference absorption changes obtained from the titration of protein $(2.5 \mu \mathrm{M})$ with increasing concentrations of the inhibitor. Representative sets of difference spectra that were recorded are shown in the insets. Spectrophotometric titrations were carried out as described under "Experimental Procedures."

hallmark ferrous CO-bound form, supporting the assumption that the new species is a ferrous compound 2 complex in the native state. On the other hand, reduction of the ferric complexes of econazole and compounds $\mathbf{4}$ and $\mathbf{5}$ resulted in the formation of a completely denaturated, partially denatured, and native ferrous ligand-free species, respectively (supplemental Fig. S2 and Table S1). The spectral identification is based on a previous stoppedflow study of the reduction of the ferric ligand-free CYP130 by sodium dithionite (20) and is further supported by their different ferrous CO species (supplemental Fig. S2 and Table S1). Also of particular interest is the failure to reduce the ferric CYP130-compound 2 complex using spinach ferredoxin and NADP +-ferredoxin reductase, indicating that the binding of this type II ligand to CYP130 decreases the redox potential of the heme.

Binding Affinity of the Arylamine Compounds-The $K_{D}$ values for the ligands were obtained from the spectral titration curves (Fig. 2) and are summarized in Table 2. For comparison, the $K_{D}$ values for econazole and clotrimazole obtained previously (8) are also listed. The sigmoid titration plots obtained for compound 2 (Fig. $2 A$ ) and compound 5 (not shown) were best-fitted to the Hill equation (Equation 3) with coefficients of 1.83 and 1.31, respectively, indicating binding cooperativity, as reported previously for the binding of econazole and clotrimazole (8). In contrast, the titration curve for compound 4 (Fig. $2 B$ ) was fitted with a rectangular hyperbola (Equation 1), indicating the absence of cooperativity, even though the active site can accommodate two molecules of this ligand (see below). Collectively, the binding affinities of all the tested arylamines were in the low micromolar range and comparable with those of the antifungal azole drugs (Table 2).

The binding of heterocyclic arylamines to CYP130 was specific. Among all of the compounds tested only compound 2 bound to CYP142 with a higher affinity than to CYP130 (Table 2). Co-crystallization of CYP142 with compound 2 or other compounds has so far been unsuccessful. No significant binding of the arylamine compounds to two other characterized M. tuberculosis $\mathrm{P} 450$ enzymes, CYP125 and CYP51, was detected. 
CYP130 in Complex with Heterocyclic Amines-Co-crystal structures were determined for compounds 2 and $\mathbf{4}$ by x-ray crystallography (Fig. 3), but co-crystals for compounds $\mathbf{3}$ and $\mathbf{5}$ were unsuitable for data collection. No significant conformational differences in the polypeptide chain associated with the binding of the heterocyclic amines were observed. Overall root

\section{TABLE 2}

The values of dissociation constants $\left(K_{D}, \mu \mathrm{M}\right)$ and Hill coefficients $(n$, if applicable) for the tested compounds

Compounds bind via a type II mechanism unless specified otherwise.

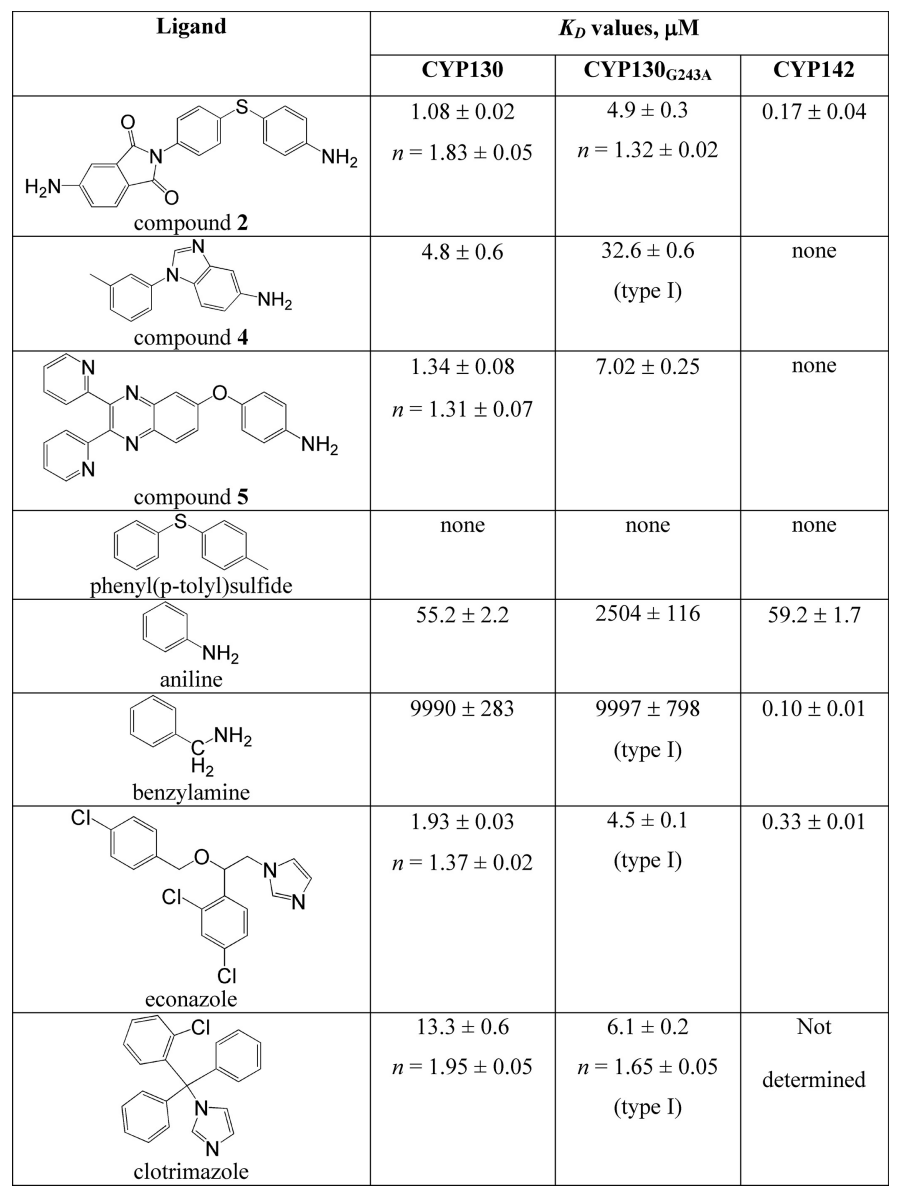
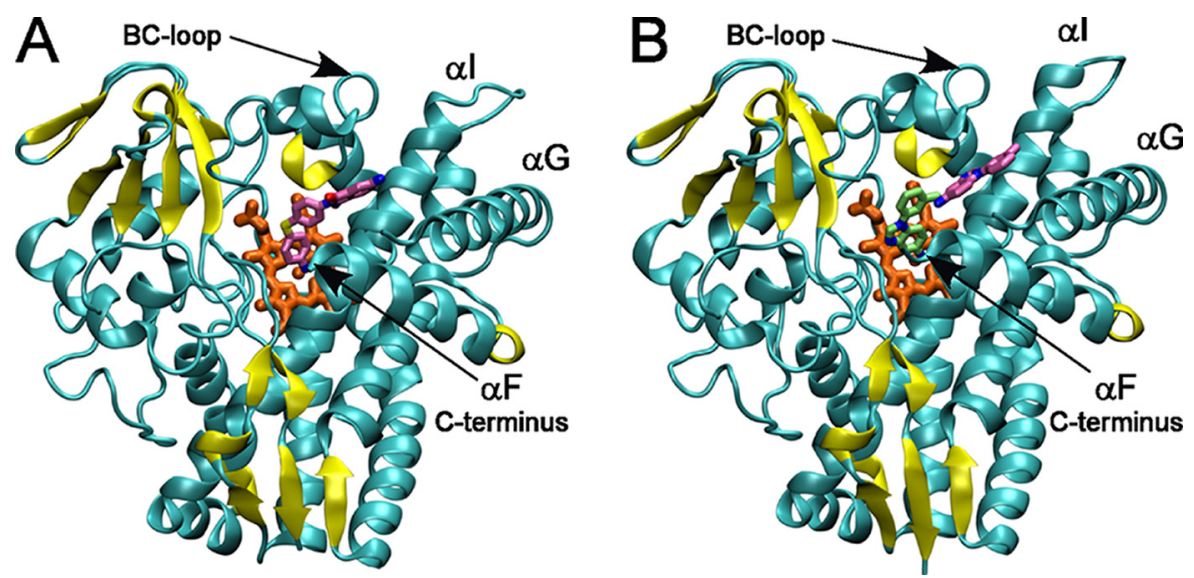

FIGURE 3. Overall view of CYP130 with ligands bound in the active site. $A$, compound $\mathbf{2}$ is highlighted in pink. $B$, two molecules of compound $\mathbf{4}$ are highlighted in lime and pink. Protein is shown as a ribbon with the $\beta$-sheets highlighted in yellow, and heme is in orange. Images were generated using the Visual Molecular Dynamics (VMD) program (28). mean square deviations compared with the ligand-free protein were within $0.47 \AA$ for the CYP130-compound 2 complex and $0.26 \AA$ for the CYP130-compound 4 complex. Some conformational differences were evident at the $\mathrm{C}$ terminus of the F-helix and in the BC-loop, which are among the inherently flexible and mobile structural elements in the $\mathrm{P} 450$ protein fold.

Although two amino groups are present in the chemical structure of compound $\mathbf{2}$ (Table 2), this ligand binds in a single preferred orientation that places the aminophenylthio group in the Fe-coordinating position. In all four molecules in the asymmetric unit, electron density was well defined for the ligating aminophenylthio moiety but scattered for the non-ligating phenyl isoindole moiety (Fig. $4 A$ ). An angular geometry of compound $\mathbf{2}$ was clearly indicated by the curvature of the electron density map bending at the thio group connecting the aminophenyl ring to the phenyl isoindole-1,3-dione moiety. Bending served to avoid a clash with the active site residues $\mathrm{Leu}^{71}, \mathrm{Val}^{90}$, and $\mathrm{Leu}^{293}$ as compound $\mathbf{2}$ spiraled around the I-helix with the isoindole ring nearly co-planar to the $\mathrm{Phe}^{238}-\mathrm{Thr}^{239}$ fragment of the I-helix backbone and the Phe ${ }^{238}$ side chain. Other hydrophobic contacts within $4 \AA$ of the isoindole ring included Pro ${ }^{88}$ and $\mathrm{Met}^{91}$ in the BC-loop.

Compound 4 binds via a single benzoimidazole amine group. Again, electron density around the heme clearly indicated the direction of Fe-coordination but is progressively less defined toward the non-ligating tolyl end of the molecule (Fig. $4 B$, molecule 4-1, cyan). A second molecule of compound 4 (Fig. 4B, molecule 4-2, pink) was fitted into the scattered electron density observed within the binding space delineated by compound 2. This molecule binds through hydrophobic interactions.

In both structurally characterized complexes the ligands bound to the heme iron via the nitrogen lone pair of electrons in a sp $^{3}$ atomic orbital of the primary arylamine. The coordination bond length for compounds 2 and 4 varied from 2.09 to $2.15 \AA$. The tetrahedral orientations of the $\mathrm{sp}^{3}$ orbitals allowed the arylamine group to approach the heme macrocycle at an angle of $\sim 30$ to $45^{\circ}$ (Fig. $5, A$ and $B$ ), in contrast to the aromatic nitrogen heterocyclic compounds, including azoles, that bind orthogonally via an $\mathrm{sp}^{2}$-hybridized aromatic nitrogen atom (Fig. $5 \mathrm{C}$ ). Furthermore, the binding of arylamines is facilitated by hydrogen bonding of one of the amine protons to the $\mathrm{Gly}^{243}$ carbonyl group (H-bond length varied between 2.73 and $2.88 \AA$ ) (Fig. 5), an interaction that alleviates the accumulation of positive charge on the nitrogen coordinated to the iron atom.

Structural Basis for Binding Cooperativity-As only one molecule of compound $\mathbf{2}$ was found in the active site in the co-crystal structure, we attribute binding cooperativity to intermolecular interactions. A similar phenomenon was described previously for the CYP130-econazole complex, where binding cooperativity re- 
$\mathbf{A}$
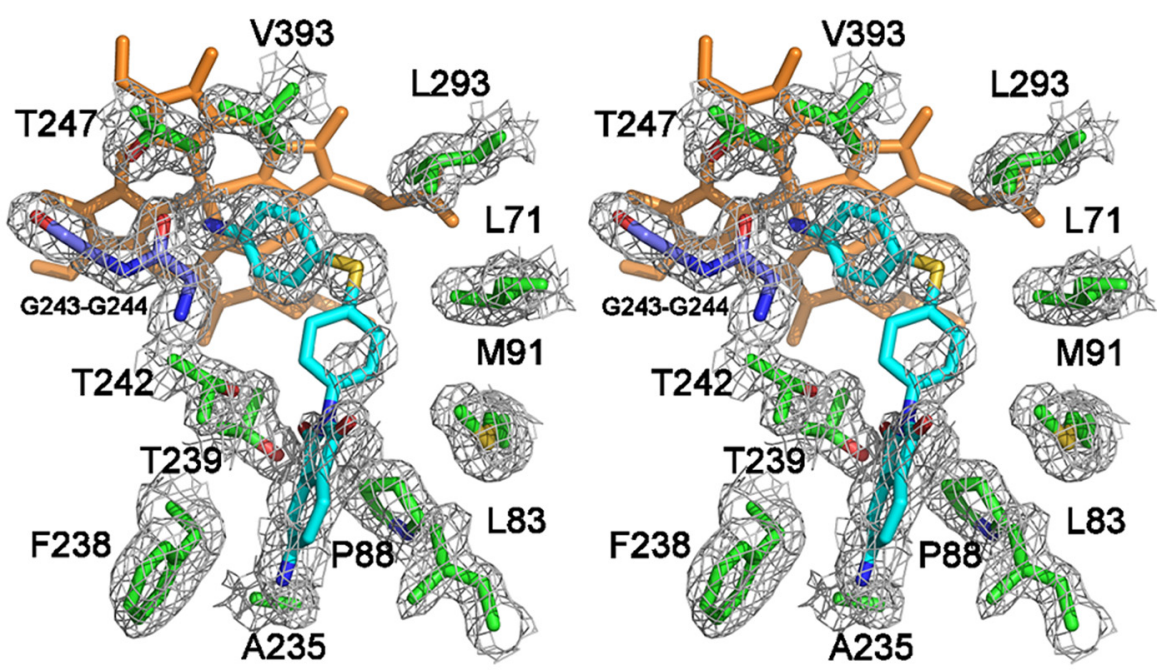

B
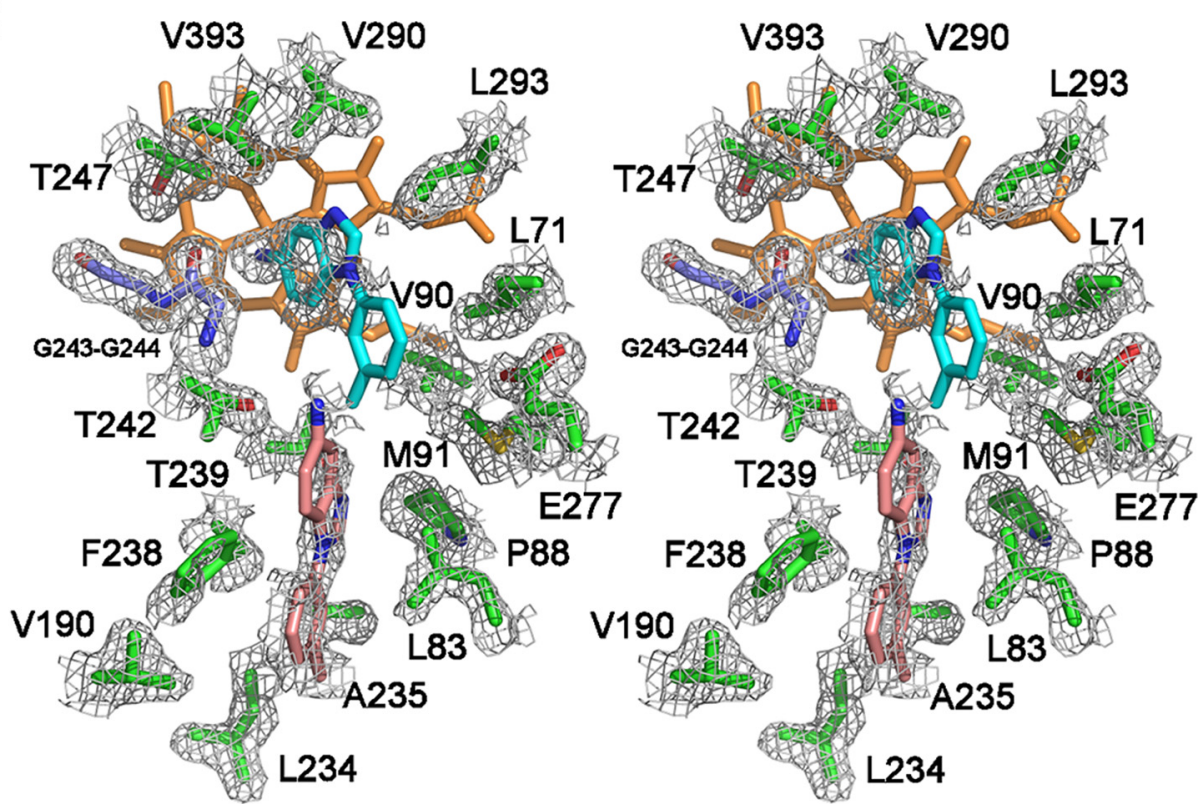

FIGURE 4. Stereo view of compounds $\mathbf{2}(\boldsymbol{A})$ and $\mathbf{4}(\boldsymbol{B})$ in the active site of CYP130. $A$, the electron densities (gray mesh) of compound $\mathbf{2}$ (cyan) and the active site residues (green) located within $5 \AA$ of the ligand are represented by a fragment of a $2 F_{o}-F_{c}$ composite omit map, where $F_{o}$ is the observed structure factor and $F_{c}$ is the calculated structure factor contoured at $1.0 \sigma . B$, the first molecule of compound 4 is cyan, and the second molecule is pink. The fragment of the I-helix comprising consecutive glycine residues 243-244 is blue. Fragments of the electron density are shown in gray mesh. Images were generated using PYMOL (29).
Ligand Binding to CYP130 The Gly $^{243}$-Gly ${ }^{244}$ motif in the middle of the I-helix is not rare among P450 enzymes, although the AlaGly pair is more common. Three M. tuberculosis P450 enzymes, specifically CYP130, CYP141, and CYP142, possess the Gly-Gly motif (Fig. 6), which appears to sterically favor angular coordination of the arylamines in CYP130. To investigate the role of an alanine versus glycine at this site, we replaced Gly ${ }^{243}$ with an alanine in CYP130 via sitedirected mutagenesis. The electronic absorption spectrum of the CYP130 ${ }_{\text {G243A }}$ mutant in the ferric resting state showed a slight red shift of the Soret band relative to the wild-type protein with a higher molar extinction coefficient (supplemental Fig. S3 and Table S2), suggesting an enhanced low-spin character for the CYP130 ${ }_{\mathrm{G} 243 \mathrm{~A}}$ mutant in the ferric resting state. The Fe-O coordination bond and $\mathrm{H}$-bond distances are consistent with this trend with the $\mathrm{Fe}-\mathrm{O}$ bond being slightly shorter in the CYP130 ${ }_{\text {G243A }}$ crystals (2.20 versus $2.23 \AA$ in the wild type) and the $\mathrm{H}$-bond to the carbonyl oxygen of $\mathrm{Ala}^{243}$ slightly longer (2.65 versus $2.62 \AA$ A) (Fig. 7). However, the absolute differences do not exceed 0.03 $\AA$, which is only three times the bond length root-mean-square deviation of $0.009 \AA$ (Table 1 and Ref 8 ), and therefore are at the borderline of credibility. The overall geometry of binding and temperature factors (B-factors) of the axial water ligand were unaffected by the G243A substitution.

The G243A substitution affects sulted from CYP130 dimerization upon econazole binding manifested in the repacking of protein molecules in the crystal lattice (8). Although the small amplitude of the conformational changes observed in the CYP130-compound 2 complex is in notable contrast with those that occur on econazole binding, compound 2 also affected CYP130 packing in the crystal resulting in loss of crystallographic symmetry and changes in the space group, unit cell dimensions, and number of molecules per asymmetric unit (Table 1). These changes in the intermolecular contacts may impact the binding affinity of the ligand resulting in binding cooperativity. It is worth mentioning that no changes were observed in the crystal parameters for the CYP130-compound $\mathbf{4}$ complex compared with the ligand-free CYP130 (8). the binding of both the arylamine and azole compounds (Table 2). The binding affinities of compounds $\mathbf{2}$ and $\mathbf{5}$ were reduced by factors of 4.5 and 5.2 , respectively. The titration plot obtained for these two compounds revealed that the cooperativity effect is also significantly reduced for compound $\mathbf{2}$ (Fig. $2 B$ ), as indicated by a smaller Hill coefficient value of 1.32 , and is totally abolished for compound 5 (data not shown). The binding affinity of compound 4 was not only 6.8 -fold reduced, but its binding mode reversed from type II to type I, as were the binding modes of econazole and clotrimazole, both with surprisingly small losses in binding affinity. Given our multiple unsuccessful attempts to obtain the pentacoordinated high-spin state of CYP130, the ease of this transition in $\mathrm{CYP} 130_{\mathrm{G} 243 \mathrm{~A}}$ was sur- 
A

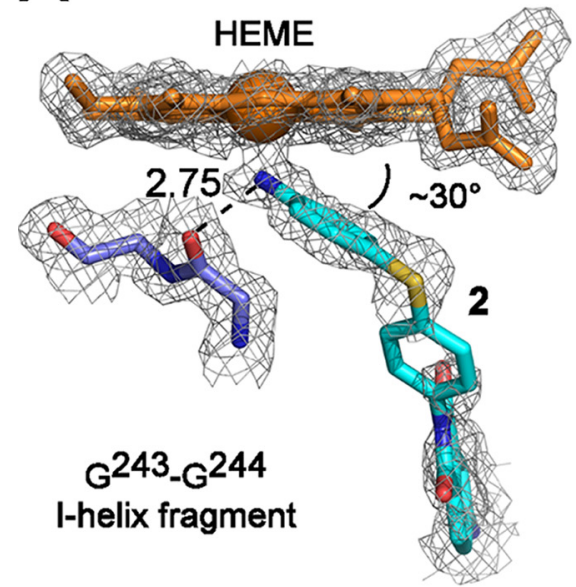

B

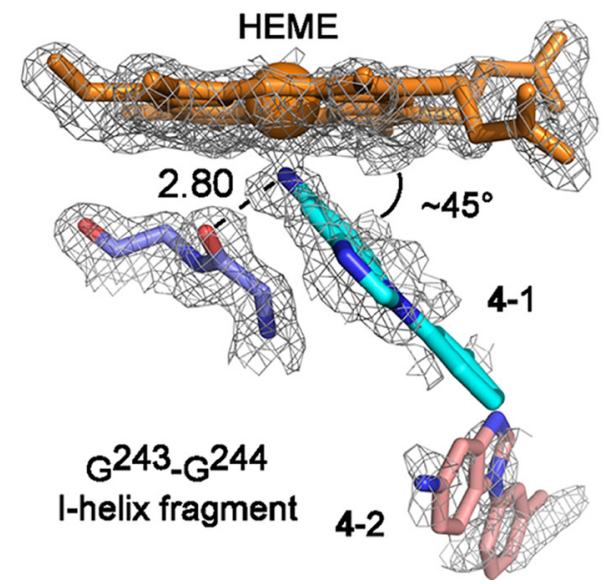

C

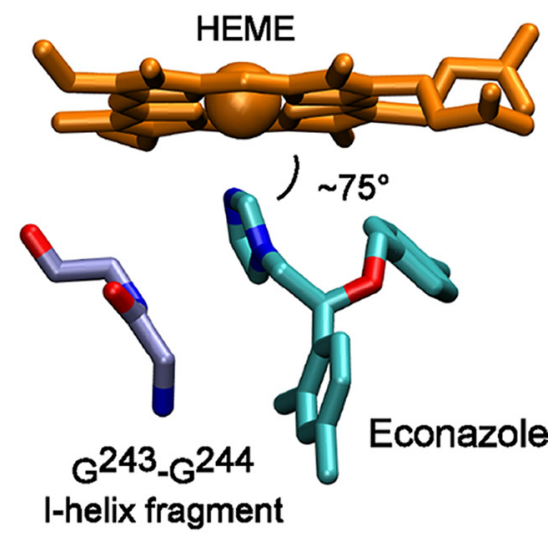

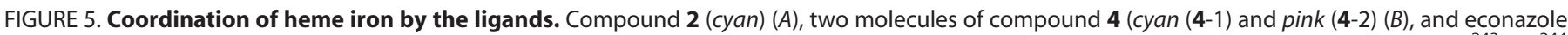

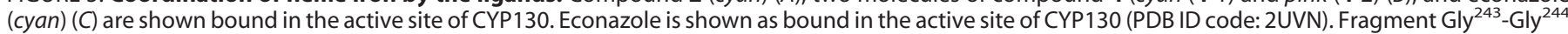

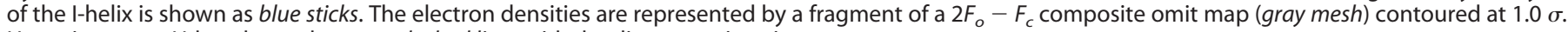
Heme is orange. $\mathrm{H}$-bonds are shown as dashed lines with the distances given in angstroms.

cyp130_Q11062
cyp123_A4KF82
cyp124_A4KJ33
cyp125_A4KM32
cyp126_A4KF94
cyp142_A4KM07
cyp135A1_A4KE19
cyp135B1_A4KEQ1
cyp137_A4KMF2
cyp138_A4KNG0
cyp128_CAB00967
cyp144_A4KHT5
cyp140_A4KI25
cyp143_A4KHU1
cyp141_A4KI22
cyp139_A4KHI4
cyp132_A4KGV5
cyp136_A4KKW2
cyp121_NP216792
cyp51_A4KF80

blelebelebelel 230 240 le 250 DTAGTLSILAFTETMVTGRDTVTGMLGGSP LIHR DRLSDQE IMAF LE LMV I A EN T T TKLLANAVYWAAH ERLSSREIASFEILIVVAGNETTRNA ITHGVLALSR EKLSDDEF GF F VVMLAVIAGNET TRNS I TQGMMAFAE PALSDAELYLFF LLF SAGAETTRNSIAGGLLALAE ERLSDDELVME T L L I L I GGDET TRH TLS G T EQLLR KP L T D E LRD L I TLLLAGHET TAAALSWTLWELA H RTMTERELRDQLITLLVAGHDTTATGLSWALERITR EQLSDSEIRDD L I TLVLAGHET TAT T LAWAF D L L L H S IMSRKD I GDELLTLLAAGHETTAATLGAFERILR . RLSDDELFFEAVLLLVIAGYSTAHMISTLELTAD GELDTLTAQVMMVTLFAAGESTAALLSAVWILA T TQLDETELRA I A G LVLVAGF ET TVN L L GNG I RMLLD . ISEIEVLGLSHLIILAGLDTVTAAVGF S LEIAR STMTDEQVVGMLLTVVIGGVDTP IAVITNGLASILH T T L SDNEIRDS IVSII TAGYET T S GA LAWA I YALL T . IWPRQRVRDEALTEMLAGHETTANAMSWFWYLMAL NRE SDAD IVNHMIE LMMAAHDISTSTAT TMAYQLAA SHVSDELFATIGVTEFGAGVIST G S L T TALISIIQ P RE SADEITGMF I SMMF A GH H T S T IASWT L I ELMR

FIGURE 6. Alignments of the I-helix in M. tuberculosis P450. Fragments of the multiple sequence alignments of the 20 P450 proteins in M. tuberculosis representing the I-helix are shown. The secondary structure annotation and residue numbering at the top correspond to CYP130. The spiral represents the $\alpha$-helix with the gap indicating disruption of the $\alpha$-helix hydrogen bonding pattern at the conserved Ala(Gly) ${ }^{243}$-Gly ${ }^{244}$ motif (highlighted in bold). Accession numbers of the proteins in the Swiss-Prot/TrEMBL (us.expasy.org/sprot) or NCBI (www.ncbi.nlm.nih.gov) data bases are given next to the name of the protein. Alignments were performed using the MAP algorithm as implemented in the BCM Search Launcher (30). The figure was generated using ESPript (31).

prising and must be due to the hydrophobic effect of the alanine side chain. Consistent with increased hydrophobicity, three water molecules $(\mathrm{HOH}-2187, \mathrm{HOH}-2292$, and $\mathrm{HOH}-2293)$ in the vicinity of $\mathrm{Gly}^{243}$ in the CYP130 wild-type (PDB ID code: 2UUG) were lost from CYP130 ${ }_{\mathrm{G} 243 \mathrm{~A}}$ (Fig. 7).

Binding of Aniline and Analogs-The contribution of the arylamine group to the binding affinity of compound 2 was estimated by examining the binding of the prototypic arylamine aniline and related compounds to CYP130 and the CYP130 $_{\text {G243A }}$ mutant (Table 2). The binding affinity of aniline to CYP130 was $\sim 55 \mu \mathrm{M}$, which is $10-20$ times stronger than reported for the hepatic microsomal fraction of P450 enzymes in earlier studies (21). The affinity of aniline dropped to $2.5 \mathrm{~mm}$ ( $\sim 50$-fold) in CYP130 ${ }_{\mathrm{G} 243 \mathrm{~A}}$, which may be due to the steric effect of the alanine side chain that interferes with both the coordination bond to the heme iron and the H-bond to the carbonyl oxygen.

Insertion of a methylene unit between the aromatic ring and the amine group resulted in a 200-fold drop of benzylamine affinity toward both CYP130 and CYP130 ${ }_{\text {G243A }}$ compared with that of aniline, together with a reversal of the benzylamine binding mode from type II to type I in CYP130 ${ }_{\mathrm{G} 243 \mathrm{~A}}$. According to the crystal structure, steric constraints imposed by the residues $\mathrm{Leu}^{71}, \mathrm{Val}^{90}$, and Leu ${ }^{293}$ contribute to the drop in the binding affinity for benzylamine versus aniline. Also, insertion of the methylene unit increases the $\mathrm{p} K_{a}$ of the amine group from 4.6 in aniline to 9.4 in benzylamine, resulting in protonation of the latter at neutral $\mathrm{pH}$ in aqueous solutions. Although the general acid-base theory does not hold in the dielectric environment of the proteins, where the $\mathrm{p} K_{a}$ of functional groups is determined largely by interactions with the surrounding groups, the apparent concentration of the active unionized form of benzylamine in solution will be strongly reduced by the protonation.

Unlike aniline, phenyl $p$-tolyl sulfide, having the angular architecture of compound 2 but lacking both the amine group and isoindole-1,3-dione moiety, did not cause any perturbation 


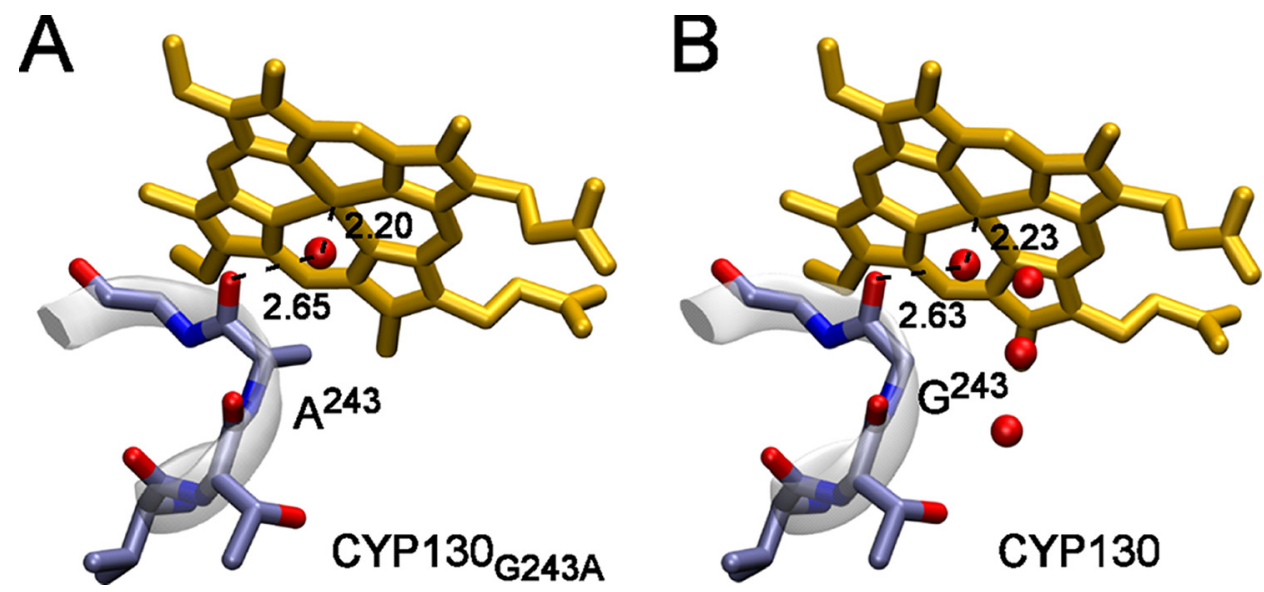

FIGURE 7. Binding of the water molecules in the active site of CYP130 and CYP130 ${ }_{\mathrm{G} 243 \mathrm{~A}}$. A more hydrophobic environment due to the side chain of $\mathrm{Ala}^{243}(A)$ results in the loss of three water molecules from the active site, which are present in the wild-type CYP130 (PDB ID code: 2UUQ) (B). The heme is in yellow, the water molecules are shown as red spheres, the 243-344 fragment of the I-helix is shown as a stick representation (blue), and the ribbon in gray. Distances between the atoms are given in angstroms.

in the Soret region of the optical spectra. Although this molecule cannot bind via a type II mechanism, one could expect type I perturbations in the optical spectra if the axial water ligand were displaced.

Interaction of CYP130 with the Nitro Analog of Compound 2The nitro analog of compound 2, 2-(4-(4-nitrophenylthio)phenyl)isoindole-1,3-dione, in which the amino group coordinating to the heme iron is substituted by a non-coordinating nitro group (supplemental Fig. S1B), also did not perturb the Soret band nor did it compete with the weak ligand imidazole for binding in the CYP130 active site (not shown). Upon enzymatic reduction of the CYP130-nitro analog mixture by the spinach ferredoxin/NADP ${ }^{+}$-ferredoxin reductase system under strict anaerobic conditions, a slow transition from the resting ferric ligand-free form to a ferric arylamine-bound form was observed (supplemental Fig. S4). In contrast to the liver microsome P450 preparations under similar anaerobic conditions (22-24), no formation of the nitrosoarene intermediate with a spectral feature at $454 \mathrm{~nm}$ has been detected. A control experiment, in which the nitro compound was preincubated with spinach ferredoxin/NADP ${ }^{+}$-ferredoxin reductase and NADPH for $60 \mathrm{~min}$ prior to the addition of ferric CYP130, confirmed that the formation of the amine form was due to the action of CYP130. Lack of nitrosoarene intermediate detection may suggest either low affinity of the latter to CYP130 or its fast conversion to the hydroxylamine and the amine forms.

\section{DISCUSSION}

The pharmacological potential of M. tuberculosis $\mathrm{P} 450$ enzymes motivated us to further explore the topology of the CYP130 active site by screening a library of synthetic organic molecules against this protein. In this screen, we identified inhibitors capable of specific binding to CYP130 via a type II mechanism that involves replacing the axial water ligand by a primary arylamine group. Coordination via the arylamine allowed compounds to approach the heme macrocycle at a sharp angle, avoiding steric constrains imposed by the I-helix that lies close to the heme plane, in contrast to the orthogonal binding of the aromatic amines (Fig. 5).
CYP130, as well as two other M. tuberculosis P450 enzymes, CYP141 and CYP142, has two consecutive glycine residues, $\mathrm{Gly}^{243}$ $\mathrm{Gly}^{244}$, on the heme-facing surface of the I-helix (Fig. 6), which favors close contacts between the I-helix and the heme macrocycle. In the ferric resting state, the carbonyl oxygen of Gly ${ }^{243}$ hydrogen bonds to the iron axial water ligand (8). This interaction is conserved across the $\mathrm{P} 450$ protein family, as judged from the conservation of the Ala(Gly)Gly motif and the crystal structure analysis of multiple representatives of the $\mathrm{P} 450$ protein family.

Spectroscopic binding studies conducted on the CYP130 $13243 \mathrm{~A}$ mutant in this work revealed that the alanine side chain substituted for $\mathrm{Gly}^{243}$ interfered with the coordination of azoles and some arylamines, reversing their binding modes from type II to type I (Table 2). Facilitated release of the axial water ligand in CYP130 ${ }_{\mathrm{G} 243 \mathrm{~A}}$ is attributed to the increased hydrophobicity of the oxygen-scission site due to the added methyl group. This difference suggests a role for the conserved Ala(Gly)-Gly motif in modulating the binding of the axial water. The wild-type CYP130 has a more hydrophilic Gly-Gly motif and tends to retain the axial water ligand, whereas the Ala-Gly variant, CYP130 ${ }_{\mathrm{G} 243 \mathrm{~A}}$, more readily releases the axial water on binding of hydrophobic ligands.

Remarkably, the binding affinities of econazole and clotrimazole are virtually independent of the differential binding mode in the wild type and mutant, suggesting that the coordination bond between the azole and the heme iron was already impaired in the wild type and was lost entirely in the CYP130 $_{\text {G243A }}$ mutant. This assumption is consistent with a previous report on steric hindrance, in which the azole drug fluconazole was forced to adopt alternative binding modes in the CYP121-fluconazole complex (25). In wild-type CYP130, econazole binds with distorted geometry (Fig. 5) by pushing $\mathrm{Gly}^{243}$ away to create an additional space (8). In CYP130 ${ }_{\mathrm{G} 243 \mathrm{~A}}$, the steric bulk of the alanine side chain created even more steric constraints, which interfered with the orthogonal coordination of the azoles and attenuate the affinity of heterocyclic amines.

Arylamines and heterocyclic arylamines are widespread in the environment as industrial chemicals, food additives, and drugs. They are notorious because $N$-oxidation by human liver cytochrome P450 enzymes converts them into mutagenic and carcinogenic products. Based on a wealth of experimental data accumulated for the mammalian P450 enzymes, structurally diverse primary alkyl-and arylamines show the entire spectrum of binding modes, ranging from type I to reverse type I to type II, with binding affinities for type II ligands (from $5 \mu \mathrm{M}$ to $7 \mathrm{mM}$ ) on average being considerably lower than for type I (from 0.1 to $500 \mu \mathrm{M})(26)$. Type I binding is imperative for catalytic conversion by $\mathrm{P} 450$. The diversity of binding modes suggests that multiple factors govern access, binding, and orientation of primary 


\section{Role of Ala(Gly)-Gly Motif in the I-Helix}

amines within the $\mathrm{P} 450$ active site. The characterization of heterocyclic arylamine binding reported here shows that the synergism of Fe-coordination, hydrogen bonding, hydrophobic interactions, and differential active site geometry all single out a compound for strong inhibitory potential toward the P450 target.

Our data suggest that compounds angularly coordinating to the heme iron have potential utility as selective inhibitors of some members of the subgroup of P450 enzymes with a Gly-Gly motif in the I-helix and should be investigated further for their antimicrobial potential, with the proviso that the arylamine functionality must be used with caution because of the incidence of idiosyncratic toxicities associated with older drugs of this category (27).

Acknowledgments - We thank Potter Wickware for critical reading of the manuscript; Petrea Kells for excellent technical support; Chris Waddling for assistance with software and instrumentation; and Chiung-Kuang Chen and the staff members of Beamline 8.3.1, James Holton, George Meigs, and Jane Tanamachi (Advanced Light Source at Lawrence Berkeley National Laboratory) for assistance with data collection. The Advanced Light Source is supported by the Director, Office of Science, Office of Basic Energy Sciences of the U. S. Department of Energy under Contract DE-AC02-05CH11231.

\section{REFERENCES}

1. Recchi, C., Sclavi, B., Rauzier, J., Gicquel, B., and Reyrat, J. M. (2003) J. Biol. Chem. 278, 33763-33773

2. Chang, J. C., Harik, N. S., Liao, R. P., and Sherman, D. R. (2007) J. Infect. Dis. 196, 788-795

3. Sassetti, C. M., and Rubin, E. J. (2003) Proc. Natl. Acad. Sci. U.S.A. 100, 12989-12994

4. McLean, K. J., Carroll, P., Lewis, D. G., Dunford, A. J., Seward, H. E., Neeli, R., Cheesman, M. R., Marsollier, L., Douglas, P., Smith, W. E., Rosenkrands, I., Cole, S. T., Leys, D., Parish, T., and Munro, A. W. (2008) J. Biol. Chem. 283, 33406-33416

5. Belin, P., Le Du, M. H., Fielding, A., Lequin, O., Jacquet, M., Charbonnier, J. B., Lecoq, A., Thai, R., Courçcon, M., Masson, C., Dugave, C., Genet, R., Pernodet, J. L., and Gondry, M. (2009) Proc. Natl. Acad. Sci. U.S.A. 106, $7426-7431$

6. Sheehan, D. J., Hitchcock, C. A., and Sibley, C. M. (1999) Clin. Microbiol. Rev. 12, 40-79

7. McLean, K. J., Cheesman, M. R., Rivers, S. L., Richmond, A., Leys, D., Chapman, S. K., Reid, G. A., Price, N. C., Kelly, S. M., Clarkson, J., Smith, W. E., and Munro, A. W. (2002) J. Inorg. Biochem. 91, 527-541

8. Ouellet, H., Podust, L. M., and de Montellano, P. R. (2008) J. Biol. Chem.
283, 5069-5080

9. Ahmad, Z., Sharma, S., and Khuller, G. K. (2005) FEMS Microbiol. Lett. 251, 19-22

10. Ahmad, Z., Sharma, S., Khuller, G. K., Singh, P., Faujdar, J., and Katoch, V. M. (2006) Int. J. Antimicrob. Agents 28, 543-544

11. Ahmad, Z., Sharma, S., and Khuller, G. K. (2006) FEMS Microbiol. Lett. 261, 181-186

12. Ahmad, Z., Sharma, S., and Khuller, G. K. (2006) FEMS Microbiol. Lett. 258, 200-203

13. Banfi, E., Scialino, G., Zampieri, D., Mamolo, M. G., Vio, L., Ferrone, M., Fermeglia, M., Paneni, M. S., and Pricl, S. (2006) J. Antimicrob. Chemother. $58,76-84$

14. Byrne, S. T., Denkin, S. M., Gu, P., Nuermberger, E., and Zhang, Y. (2007) J. Med. Microbiol. 56, 1047-1051

15. Podust, L. M., von Kries, J. P., Nasser Eddine, A., Kim, Y., Yermalitskaya, L. V., Kuehne, R., Ouellet, H., Warrier, T., Alteköster, M., Lee, J. S., Rademann, J., Oschkinat, H., Kaufmann, S. H., and Waterman, M. R. (2007) Antimicrob. Agents Chemother. 51, 3915-3923

16. Chen, C. K., Doyle, P. S., Yermalitskaya, L. V., Mackey, Z. B., Ang, K. K., McKerrow, J. H., and Podust, L. M. (2009) PLoS Negl. Trop. Dis. 3, e372

17. Schenkman, J. B., Remmer, H., and Estabrook, R. W. (1967) Mol. Pharmacol. 3, 113-123

18. Leslie, A. G. W. (1992) Joint CCP4-ESF-EAMCB Newsletter on Protein Crystallography, Vol. 26, Daresbury Laboratory, Warrington, UK

19. Otwinowski, Z., and Minor, W. (1997) Methods Enzymol. 276, 307-326

20. Ouellet, H., Lang, J., Couture, M., and Ortiz de Montellano, P. R. (2009) Biochemistry 48, 863-872

21. Remmer, H., Schenkman, J., Estabrook, R. W., Sasame, H., Gillette, J., Narasimhulu, S., Cooper, D. Y., and Rosenthal, O. (1966) Mol. Pharmacol. 2, 187-190

22. Keilin, D., and Hartree, E. F. (1943) Nature 151, 390-391

23. Franklin, M. R. (1976) Chem. Biol. Interact. 14, 337-346

24. Mansuy, D., Beaune, P., Cresteil, T., Bacot, C., Chottard, J. C., and Gans, P. (1978) Eur. J. Biochem. 86, 573-579

25. Seward, H. E., Roujeinikova, A., McLean, K. J., Munro, A. W., and Leys, D. (2006) J. Biol. Chem. 281, 39437-39443

26. Hlavica, P. (2006) Biochim. Biophys. Acta 1764, 645-670

27. Kim, D., and Guengerich, F. P. (2005) Annu. Rev. Pharmacol. Toxicol. 45, $27-49$

28. Humphrey, W., Dalke, A., and Schulten, K. (1996) J. Mol. Graph. 14, 33-38

29. DeLano, W. L. (2002) The PyMOL Molecular Graphics System, DeLano Scientific, San Carlos, CA

30. Smith, R. F., Wiese, B. A., Wojzynski, M. K., Davison, D. B., and Worley, K. C. (1996) Genome Res. 6, 454-462

31. Gouet, P., Courcelle, E., Stuart, D. I., and Métoz, F. (1999) Bioinformatics 15, 305-308

32. Laskowski, R. A., MacArthur, M. W., Moss, D. S., and Thornton, J. M. (1993) J. Appl. Crystallogr. 26, 283-291 